\title{
Software Quality and Productivity Model for Small and Medium Enterprises
}

\author{
Jamaiah H. Yahaya \\ Faculty of Information Science and Technology, \\ Universiti Kebangsaan Malaysia, Bangi, \\ Selangor, Malaysia \\ Asadullah Tareen \\ Ghazni University, \\ Qala-E Jawaz, Ghazni, Afghanistan
}

\author{
Aziz Deraman \\ School of Informatics and Applied Mathematics, \\ Universiti Malaysia Terengganu, \\ Kuala Terengganu, Malaysia
}

\author{
Abdul Razak Hamdan \\ Faculty of Information Science and Technology, \\ Universiti Kebangsaan Malaysia, Bangi, Selangor, Malaysia
}

\begin{abstract}
The enterprises today including small and medium enterprises (SMEs) are dependent on software to accomplish their objectives and maintain survivability and sustainability in their businesses. Although many studies in software quality have been carried out previously, they still lack for the correlation between software quality and the impact to SMEs productivity. The objectives of this study are to determine the quality factors from management's perspective and to determine the impact of software quality and the productivity of SMEs. It is implemented through a survey conducted in Malaysia which involves 43 respondents who are among the managers and management of SME companies. The survey indicates that efficiency, expandability, functionality, reusability, safety and usability are the most influential factors from a management perspective. The research hypotheses defined are accepted with strong relationships between the defined variables. It shows that the level of software quality assessment in SMEs is correlated with the level of its productivity. Based on these findings, a software quality and productivity (SQAP) model for SME is developed. This paper presents the development of SQAP model which can be used as the standard and guideline in the process of obtaining and upgrading software in SMEs and can further be applied in quality assessment in the organisations.
\end{abstract}

Keywords-Software Quality; Small and Medium Enterprise; SME Productivity; Software Quality and Productivity Model

\section{INTRODUCTION}

The business world today is progressing and changing at a rapid pace. The fundamental reason behind the rapid progress is technological development and advancement. No matter which type of organisation, it is indispensable for them to embrace the latest technology. Small and Medium Enterprises (SMEs) are mostly defined in term of a number of employees and the annual turnover. European Commission defined SMEs criteria as: 1) the organisation is an enterprise, 2) has fewer than 250 employees, 3) has an annual turnover not exceeding $€ 50$ million, and 4) is an autonomous company [1]. Different countries define SMEs slightly different which compatibles and matches the specific country requirement and expectation. In Malaysia, the SME definition is stated as the following:

Sales turnover and a number of full-time employees are the two criteria used in determining the definition with the
“OR” basis as follows:

For the manufacturing sector, SMEs are defined as firms with sales turnover not exceeding RM50 million OR a number of full-time employees not exceeding 200.

For the services and other sectors, SMEs are defined as firms with sales turnover not exceeding RM20 million OR a number of full-time employees not exceeding 75 [2].

Today, only those organisations whether SMEs or Multinational Corporations can survive that are tech-savvy and adopt strategies to utilise technology. In this vogue of usage of technology, organisations do not only rely on software and technology tools, but also spend an enormous amount on the maintenance and quality of these tools and software products. All these strenuous efforts are made by the organisation to maximise the productivity and to achieve efficiency in every operation that they perform.

The study presented in this paper aims to discover the role of software quality on the success of SMEs. It takes into consideration the impact of software quality assessment on the productivity of SMEs. This paper is organised as: In Section 2, the research background and the related works are presented. Section 3 presents the empirical study, and Section 4 discusses the development of Software Quality and Productivity (SQAP) Model for SME. Section 5 proceeds with some discussions and finally, the work of this paper is concluded in the last section.

\section{RESEARCH BACKGROUND}

\section{A. SMEs Success and ICT}

The success or failure of SMEs is of paramount importance for the nations' economies, particularly, as the activities of many SMEs led to failure in early 1970 [3]. Various factors behind the failure of SMEs have been taken into consideration. The main failure of SMEs was observed to coincide with the frequent usage of information technology (IT). However, in recent years, success of new SMEs has rebounded to some extent, especially in the field of manufacturing, but the overall negative correlation that exists between economic success and the advent of computers is 
behind most of the arguments that IT has not aided to gain SMEs success or even some researches recount that investments of IT have been very counterproductive [4].

Cron and Sobol conducted a study to investigate the impact of IT in SME [5]. They revealed that on an average, the impact of IT on the success of SMEs was not significant, but it appeared to be linked with both the low and high performers. The findings of their study established the basis for the hypothesis that all aspects of IT such as software, technological tools and networks tend to reinforce management approaches which, in turn, helps the success of well-organised SMEs. However, those managers who are confused and are not successful in structuring the production operation in the first place may fail [6].

A study by Strassman presented the idea that there was no correlation between IT and success and returned on investment [7]. This result came from a study in which 38 SMEs and some of the top performers in this sample heavily spent on IT and some SMEs did not spend on IT.

On the other hand, the study of Panko [8] showed that SMEs that employed the latest technology updated the software and maintained the performance and quality of software were successful. Furthermore, another study done by Hamdan et al. had revealed and discovered several factors that influence the used of IT by the SMEs. The main factors discovered by this study were: increase in sales and productivity, improve internal efficiency, enrich company's image or opportunities and quality, and some other less important factors [9].

Thus, past studies showed mixed results about the correlation between usage of IT or software and it is impacted by the success and failure of SMEs. This was a controversial situation that was presented by different studies which have been conducted. Therefore, it is essential to look at this matter and explore the relationship between software quality assessment and its impact on the performance of the SMEs.

\section{B. Software Quality Models on the SMEs}

Literature has demonstrated that many software quality models have been introduced and invented but with ambiguous practicality and functionality. Literature also provides other taxonomies where four quality models namely McCall, Boehm, ISO 9126 and Gillies relational quality models are regarded as prominent and well known models with renowned popularity [10]. These models have a lot of commonalities in terms of quality factors. McCall model, Boehm model, ISO 9126 and Gillies Relational model have more or less similar quality factors and many researchers adopted these well-known fundamental models as their baseline of their works. The common quality factors in all these models are: Efficiency, Maintainability, Portability, Functionality, Reliability and Usability which have emerged as the recognised quality factors and are considered as most related to the quality of the software product [10]. These quality attributes are also referred to as the behavioural attributes of the software or quality in-use. These quality attributes are consistent with the new enhanced quality model invented [11] and embedded the impact attributes which related to human factors.

The product quality model defined in ISO/IEC 25010 is the new version of ISO 9126 model. It comprises of eight attributes: functional suitability, performance efficiency, compatibility, usability, reliability, security, maintainability and portability [12]. Each of these attributes is broken down into several sub attributes with similar structure as ISO 9126 model. These quality attributes are applicable to both computer system and software products [13].

Literature has evidenced studies in software quality assessment from development process point of views such as Capability Maturity Model (CMM), the ISO 9000 [14] [15], and SPAC model [16]. A study on SME and quality measurement elements (QME) was investigated and revealed best QMEs for a company. It listed QMEs such as product size and a number of faults detected [17]. Other studies have been reported on the software quality model from customers and user's perspectives [17] [18] [19] [20] but do not apply specific to SMEs implementation.

\section{The Management of Quality in SMEs}

Quality management ensures that organisation, product or services is consistent and meet a certain level of standard and expectation. It has four main components: quality planning, quality control, quality assurance and quality improvement [21]. Quality management focuses not only on product and service quality, but also the means to achieve it. It therefore uses quality assurance and control of processes as well as products to achieve more consistent quality.

Despite the clear benefits offered by the SMEs and their results of effective management, there are many SMEs that do not have a discerning person having adequate knowledge of these functions and, when this person exists, the majority of cases focus on the administrative aspects of the role extending to training. Previous studies revealed that experience, training, education and use of technology were necessary for employees to sustain in SMEs [21][34][35].

A study done by Foong [22] stated that the success of IT in SMEs was affected by the existence of some conditions including strong management support as the main condition. Similarly, management supports towards IT adoption could significantly participate in the IT adoption success within SMEs [23]. It was also argued that the management IT knowledge and experience were other characteristics which affected IT adoption in SMEs. Another study confirmed that SMEs with managements who were more knowledgeable regarding IT were more likely to adopt IT and greater knowledge of managements would decrease the degree of uncertainty associated with IT which would lead to lower risk of IT adoption [3].

Furthermore, Liao et al. claimed that in SMEs where managements have higher computing skills level are more pleased with the applied IT compared to those with inferior skills [24]. Such views strengthen the view that adequate knowledge of IT and its consequential influences over organisation can be stimulating and supportive for the adoption of IT in SMEs. More specifically, the management in SMEs can impact the selection and assessment of the quality 
of the software [25].

Another factor that influences software quality assessment is human resource development which entails training, formal education and experience. Human resource development and management are the critical practices for improving business and management processes [26]. Yang et al. further concluded that human resource management as total quality management (TQM) practice significantly correlated with customer satisfaction [27]. Due to the association between human resource, management and different performance measurement indicators, it can be concluded that human resource has a significant effect on the software quality, and correspondingly on SME productivity.

Training and education spread the knowledge of constant improvement and innovation in service process to achieve full benefits and business excellence. Talib [28] reported the role of training and education in upholding high quality level within the SMEs. In addition, the research on total quality management (TQM) also reported a positive correlation between training and education, and organisation performance [28]. Therefore, hypothesis attempts to find a relationship between training and education and quality performance.

\section{THE EMPIRICAL STUDY}

This study uses two types of data, the primary and secondary data. To obtain the secondary data, a comprehensive literature review was carried out. This study obtained the primary data through empirical study and data analysis. This study employed quantitative approach using a questionnaire that aimed to test the hypothesis with a large population and generalise the result. The empirical study was conducted in Klang Valley of Malaysia where the capital city of Malaysia, Kuala Lumpur is located. Klang Valley is centred in Kuala Lumpur, and includes its adjoining cities and towns in the state of Selangor.

Next, the collected data was analysed using both descriptive and inferential statistics. Furthermore, based on the relationships between research variables, a software quality and productivity (SQAP) model was developed.

\section{A. The Survey and Questionnaire Design}

This survey was conducted which involved forty-three (43) respondents from randomly selected IT companies in Malaysia. They represented at the management level in their companies. The companies were randomly selected from the list of SMEs and then the questionnaires were sent to the members of the companies through email and hard mail. The questionnaire contained fifty (50) items that consisted of five main sections: respondent background, organisation background, SME quality control, human resource development and training. The detail on the empirical study and analysis can be found in Yahaya et al. [29].

\section{B. The Software Quality Attributes}

Fifteen software quality attributes are identified from literature: efficiency, expandability, flexibility, functionality, integrity, interoperability, maintainability, portability, reusability, reliability, safety, survivability, testability, usability and verifiability [11] [30]. One of the tasks defined in the survey is to examine and assess the importance of these attributes from their perspectives. Respondents specify the importance of these attributes in Likert scales of 1 (not important) to 5 (very important).

This survey discovers that the most influential factors of software quality attributes are: efficiency, expandability, functionality, reusability, safety and usability (see Table 1). The selected software quality attributes are considered as the important attributes for measurement of quality assessment from management's perspective.

TABLE I. QUALity AtTRIBUtes: The MEAN SCORES

\begin{tabular}{|l|l|l|l|}
\hline Attributes & Mean & Attributes & Mean \\
\hline Efficiency & 4.3 & Reusability & 4.3 \\
\hline Expandability & 4.3 & Reliability & 4.1 \\
\hline Flexibility & 4.1 & Safety & 4.3 \\
\hline Functionality & 4.3 & Survivability & 4.1 \\
\hline Integrity & 4.0 & Testability & 4.1 \\
\hline Interoperability & 3.9 & Usability & 4.3 \\
\hline Maintainability & 4.0 & Verifiability & 3.9 \\
\hline Portability & 3.8 & & \\
\hline
\end{tabular}

\section{The Hypothesis Tests}

A statistical test was applied to examine the relationship between the variables based on research objectives and hypothesis. The hypotheses are as the following:

H1: There is a positive statistical relationship between SME quality control and software quality.

H2: There is a positive statistical relationship between human resource development and software quality.

H3: There is a positive statistical relationship between software quality and SME's productivity.

The result shows that there is a statistically positive with strong magnitude significant relationship between the level of SME quality control and human resource training and software quality. The result of the analysis reveals that the higher level of SME quality control and human resource training is linked to the higher level of software quality and vice versa. It means that with any increase in SME quality control and human resource development and training, the software quality will increase too. The result of the test also reveals that there is a statistically positive with strong magnitude significant correlation between software quality and SMEs productivity. This shows that any increase in quality, the productivity will increase as well. Hence, as there is a direct, positive relationship between software quality and SME productivity, the productivity of the SMEs is increased as discussed in [29].

\section{Software Quality and Cost Criteria for Selection}

In the case of quality issues, it is interesting to discover that the respondents acknowledged the importance of quality and its associated issues. In other words, the respondents considered quality issues to be extremely important in the organisation. A similar case was observed during the investigation of the importance of price in software selection where $43 \%$ of the respondents agreed that price consideration during the selection was significantly important whereas $28 \%$ of the respondents indicated that the price hold average 
important for their organisation and the remaining $19 \%$ indicated that the price is somewhat important for their organisation [29].

\section{SQAP: SOFTWARE QUALITY AND PRODUCTIVITY MODEL FOR SME}

Based on the empirical findings as discussed in previous section and literature study, the SQAP model was developed. The empirical study shows that there is a statistically positive with strong magnitude significant relationship between the level of quality control and human resource training and software quality. The result reveals that the higher level of quality control and human resource training are linked to higher level of software quality and vice versa. It indicates that with any increase in quality control and human resource training, the software quality will increase too.

The correlation test also reveals that there is a strong positive relationship between software quality and SME productivity. In other words, with any increase in software quality, the SME productivity will increase as well. Hence, as there is a direct, positive relationship between software quality and SME productivity, the productivity and efficiency of the SMEs are increased.

Software quality assessment is affected by some factors such as quality control which consists of human resource development, software quality acquisition and software quality assurance, and software quality factors. As identified in the empirical study, software quality attributes embedded in this model consist of efficiency, expandability, functionality, reusability, safety and usability. While, in human resource development aspect includes training, education and experience. In addition, there is a positive correlation between software quality and SME productivity, and any changes in the value of the mentioned factors can affect the productivity of SMEs. These factors and the correlations are demonstrated and proposed in the Software Quality and Productivity (SQAP) model as shown in Figure 1.

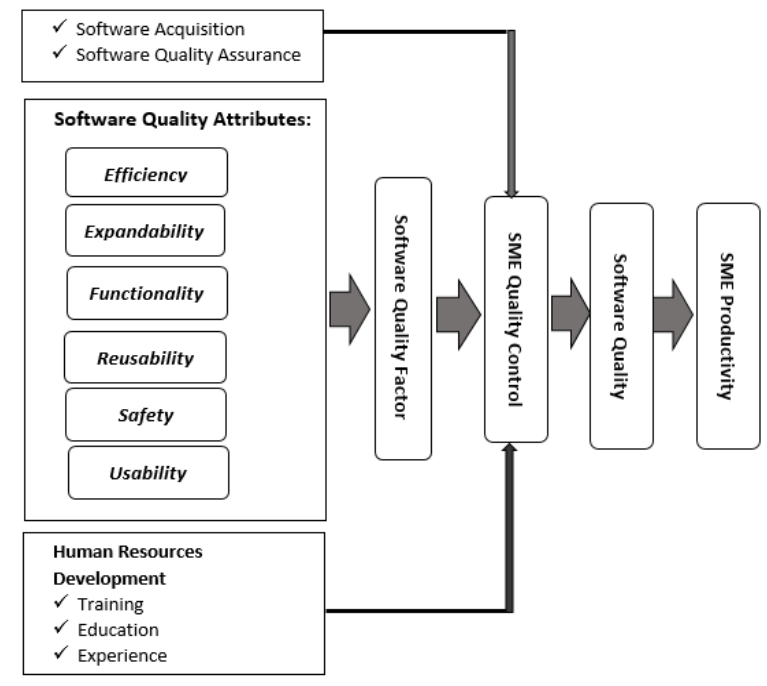

Fig. 1. Software Quality and Productivity (SQAP) Model for SME

\section{DISCUSSION}

Human resource development including training, education and experience has been highlighted as important to the software quality assessment by SMEs management. In order to develop or select appropriate software, proper training and education are required and this consistent with the previous finding [28]. Furthermore, managers should benefit from various experiences in upgrading or selecting software product in order to improve their productivity. Likewise, the importance of experience for improving software quality is also revealed [31].

Software acquisition is another factor which was considered as important for software quality assessment by SME management. So, measures need to be taken to get the managers acquire the necessary knowledge, skill and information regarding the software used in the SME. This will promote the software quality assessment process. The importance of this factor to SME productivity is also highlighted by Daneshgara [32].

Software quality assurance (SQA) factors are essential in software quality assessment. This indicates that the management confirms that the selected software product meets and complies with defined standard quality specifications. Similarly, Mishra and Mishra highlighted the importance of SQA on SME productivity [33]

Moreover, SQAP model indicates that six quality attributes have given impact to the quality of software. The attributes are efficiency, expandability, functionality, reusability, safety and usability. Thus, the management of SME should take into account these factors while upgrading or selecting software product.

For an effective software development, it is essential that the process focuses on every element associated with quality and control. Considering this, the software quality and productivity model or SQAP were developed that can be used by organisations and individuals for bringing significant improvement in software development process. For instance, for optimal software quality, the model suggests that software development should focus extensively on efficiency, reliability, integrity, expandability, portability, flexibility, maintainability and etc. On the other hand, quality can be controlled by introducing quality assurance and acquisition program. However, this cannot be done without human resource development. Our model suggests that the continuous training and development sessions should be provided to the workforce to ensure the effectiveness of software quality control. Proper training and education of employees will eventually lead to effective quality control. Once quality is ensured along with quality control, the SMEs can develop quality software, which will eventually enhance the SME's productivity.

\section{CONCLUSIONS AND FUTURE WORK}

The proposed model, SQAP, is applicable to any SMEs irrespective of type and nature of the organisation. The model will help organisations to enhance its productivity, which will eventually provide it with an opportunity to survive and thrive in the industry. SQAP focuses on certain aspects of software 
quality and productivity of SMEs. This study was implemented in Malaysia where an empirical study was conducted to obtain and verify software quality attributes, and correlation and relationships among defined variable as discussed in this paper.

Future study is recommended with more respondents from different countries on the SMEs and applied to the real case study. At the same time, the proposed model can be used for the development of a more effective model that can allow organisations in the development of profitable and optimal quality models. The strategic planning of SMEs should be directed and integrated with the proposed model which later may support the software quality program in the organisation and move actions toward continuous improvement of the software product in the short, medium and long term.

\section{ACKNOWLEDGMENT}

This research is funded partly by Malaysia Ministry of Higher Education under the Fundamental Research Grant Scheme (FRGS/1/2015/ICT04/UKM/02/1).

\section{REFERENCES}

[1] European Commission. http://ec.europa.eu/growth/smes/businessfriendly-environment/sme-definition_en, 2017.

[2] SME Corp. Malaysia, "SME definitions," https://www.smecorp.gov.my/index.php/en/policies/2015-12-21-09-0949/sme-definition, 2017.

[3] J.Y. Thong, and C.S. Yap, "CEO characteristics, organisational characteristic and information technology adoption in small businesses", Omega, vol. 23, no. 4, pp. 429-442, 1995.

[4] B. Erik, "The productivity paradox of information technology: Review and assessment," Communication of the ACM, vol. 12, 2003.

[5] W.L. Cron, and M.G. Sobol, "The relationship between computerization and performance: A strategy for maximizing the economic benefits of computerization," Journal of Information and Management, vol. 6, pp. 171-181, 2003.

[6] P. Drucker, Management. Routledge, 2012.

[7] P.A. Strassman, The Business Value of Computers, Information Economics Press, New Canaan, Conn. pp. 10-12, 2009.

[8] R. Panko, "Is Office Productivity Stagnant?" MIS Quarterly, vol. 15, no. 2, 2008.

[9] A.R. Hamdan, J.H. Yahaya, A. Deraman, and Y.Y. Jusoh, "The success factors and barriers of information technology (IT) implementation in small and medium enterprises (SMEs): An empirical study in Malaysia," International Journal of Business Information System (IJBIS), vol. 21, no. 4, pp. 477-494, 2016.

[10] A. Sharma, R. Kumar, and P. Grover, "Estimation of quality for software components - an empirical approach," ACM SIGSOFT Software Engineering Notes, vol. 3, no. 5, pp. 1-10, 2008.

[11] J.H. Yahaya, and A. Deraman, "Measuring the unmeasurable characteristics of software quality," International Journal of Advancements in Computing Technology, vol. 2, no. 4, pp. 95-106, 2010.

[12] ISO/IEC 25010, http://iso25000.com/index.php/en/iso-25000standards/iso-25010, 2017.

[13] ISO/IEC 25010:2011, Systems and software engineering -- Systems and software Quality Requirements and Evaluation (SQuaRE) -- System and software quality modelshttps://www.iso.org/standard/35733.html, 2011.

[14] B. Fitxgerald, and K. J. Stol, "Continuous software engineering and beyond: trends and challenges," In Proceedings of the 1st international workshop on rapid continuous software engineering, ACM, pp. 1-9, 2014

[15] S. Islam, H. Mouratidis, and E.R. Weippl, "An empirical study on the implementation and evaluation of a goal-driven software development risk management model," Information and Software Technology, vol. 56, no. 2, pp. 117-133, 2014.

[16] F. Baharom, J.H. Yahaya, A. Deraman, and A.R. Hamdan, "Software process certification: a practical model for maintaining software quality," IJIPM: International Journal of Information Processing and Management, vol. 4, no. 3, pp. 51-61, 2013.

[17] B. Shrestha, Best QMEs for Measurement of Software Quality for SMEs. Master thesis, Faculty of Science and Forestry, School of Computing, Univ. of Eastern Finland, 2016.

[18] G. .-J. Ahn, M. Ko, and M. Shehab, "Privacy-enhanced user-centric identity management," IEEE Communications Society (IEEE ICC) proceedings, 2009.

[19] A.B. Spantzel, J. Camenish, T. Gross, and D. Sommer, User Centricity: A Taxonomy and Open Issues, Department of Computer Science, Purdue University, IBM Zurich Research Lab, Switzerland, 2007.

[20] J.H. Yahaya, A. Deraman, A.R. Hamdan, and Y.Y. Jusoh, "Userperceived quality factors for certification model of web-based system," International Journal of Computer, Information, Mechatronics, Systems Science and Engineering, vol. 8, no. 5, pp. 576-582, 2014.

[21] L.K. Rose, N. Hoppen, and J.L. Henrique, "Management of perceptions of information technology service quality," Journal of Business Research, vol. 62, no. 9, pp. 876-882, 2009.

[22] S.Y. Foong, "Effect of end-user personal and systems attributes on computer-based information system success in Malaysian SMEs," Journal of Small Business Management, vol. 37, no. 3, pp. 81, 1999.

[23] M. Ghobakhloo, T.S. Hong, M.S. Sabouri, and N. Zulkifli, "Strategies for successful information technology adoption in small and mediumsized enterprises," Information, vol. 3, no. 1, pp. 36-67, 2012.

[24] C. Liao, P. Palvia, and J.L. Chen, "Information technology adoption behavior life cycle: Toward a technology continuance Theory (TCT)," International Journal of Information Management, vol. 29, no. 4, pp. 309-320, 2009.

[25] A. G. Valdez Menchaca, C. V. Lebrun, E. O. Benitez, J. C. Perez Garcia, O. A. Garza, O. M. Preciado Martinez, and S.R. Castaneda Alvarado, "Practical application of enterprise architecture, a study case of SME Metalmechranic in Mexico," European Scientific Journal, vol. 9 , no. 10, 2014.

[26] N. Nordin, B. Md Deros, D. Abdul Wahab, and A.N. Ab. Rahman, "A framework for organisational change management in lean manufacturing implementation," International Journal of Services and Operations Management, vol. 12, no. 1, pp. 101-117, 05/2012

[27] M.G. Yang, P. Hong, and S.B. Modi, "Impact of lean manufacturing and environmental management on business performance: An empirical study of manufacturing firms. International Journal of production economics, vol. 129, no. 2, pp. 251-261, 2011.

[28] F. Talib, Z. Rahman, and M.N. Qureshi, "The relationship between total quality management and quality performance in the service industry: a theoretical model," International Journal of Business, Management and Social Sciences, vol. 1, no. 1, pp. 113-128, 2010.

[29] J.H. Yahaya, A. Tareen, and A. Deraman, "Software quality and the success of small and medium enterprises: The management perspective," Journal of Engineering and Applied Sciences, 2017, in press, to be published.

[30] G.R. Dromey, "Cornering the chimera," IEEE Software, January, pp. 33-43, 1999.

[31] K. Schneider, Experience and Knowledge Management in Software Engineering, Springer-Verlag Berlin Heidelberg, 2009.

[32] F. Daneshgara, G.C. Low, and L. Worasinchaia, "An investigation of 'build vs. buy' decision for software acquisition by small to medium enterprises," Information and Software Technology, vol. 55, no. 10, pp. 1741-1750, 2013.

[33] A. Mishra, and D. Mishra, "Software quality assurance models in small and medium organisations: a comparison," International Journal of Information Technology and Management, vol. 5, no. 1, 2006.

[34] R. Rossi, "Quality developments in the Brazilian software industry and the relevance of strategic issues for software quality," Lecture Notes on Software Engineering, vol. 2, no. 4, 2014.

[35] OECD, Skills Development and Training in SMEs, Local Economic and Employment Development,(LEED), OECD Publishing. http://dx.doi.org/10.1787/9789264169425-en, 2013. 